\title{
Guidelines for Microanalysis Using the Energy-dispersive Spectrometer
}

\author{
P. K. Carpenter \\ Earth and Planetary Sciences, Washington University, St. Louis, MO 63130, USA
}

Energy-dispersive spectrometry (EDS) is a powerful analytical tool which is routinely used for qualitative and quantitative analysis as well as compositional mapping. Qualitative analysis is used to establish the inventory of elements and frequently the relative change in X-ray intensity is diagnostic for the material being analyzed, and quantitative analysis is used to measure the elemental concentrations. Both activities benefit from an understanding of sample preparation, X-ray physics, the EDS measurement process, and conversion of measured X-ray intensities to concentration via the ZAF correction [1]. The following outlines key points for the technologist to be aware of when doing EDS analysis.

The EDS analysis system should be properly calibrated so that observed x-ray peaks are at their expected energies and low energy photos are detected. Different pulse processing settings are used for low vs. high count rates and the high resolution setting should be used for quantitative analysis while mapping should use a lower resolution (high throughput) setting. The silicon-drift (SDD) EDS represents a significant advance with excellent performance for many applications. The user should be alert to coincident pulse artifacts as they are a by-product of high count rate applications using the SDD. These pileup peaks can be mistaken for elements not in the sample and their identification is the primary challenge for the analyst. Possible gain shift and peak resolution changes at high count rate can also negatively affect quantitative analysis, so attention to the probe current ("spot size") and the use of a standard probe current are desirable.

The sample should be flat, micropolished, coated with carbon, and analyzed at the same x-ray takeoff angle used for the standards. Rough or tilted surfaces should be avoided because the effective x-ray takeoff angle is at best poorly known. While it is possible to use the tilting stage of an SEM to place a tilted sample in the horizontal orientation for analysis, sample orientation is not well constrained in an SEM, and therefore the takeoff angle deviates from an assumed value. The x-rays of light elements are strongly absorbed and it is important to understand the effect of roughness and sample orientation.

Modern EDS software packages include quantitative analysis and spectrum imaging (SI) capabilities. The EDS peak measurement and ZAF correction form the two parts of quantitative analysis. Data collected in SI mode typically stores the complete EDS spectrum at each point in the image for processing, and is used for chemical phase identification via software similar to principle component analysis, as well as quantitative analysis. This couples imaging and chemical analysis and is one of the best features of EDS systems for the analyst. In conventional quantitative analysis, the background-subtracted x-ray peak intensity measured on the sample is divided by the same measurement on the standard; this is the k-ratio $\mathrm{k}=(\mathrm{P}-\mathrm{B})^{\mathrm{sample}} /(\mathrm{P}-\mathrm{B})^{\text {standard }}$, and the concentration $\mathrm{C}$ for a given element is calculated from the ZAF correction using $\mathrm{C}=\mathrm{k} * \mathrm{ZAF}$, where the corrections for $\mathrm{Z}$ (atomic number), A (characteristic X-ray absorption), and $\mathrm{F}$ (characteristic X-ray fluorescence) are combined for presentation in typical output as "ZAF". Peak intensities from EDS spectra are typically calculated by least-squares fitting of sample spectra using standard peaks that have been measured by the user or have been previously measured and stored for use as "standardless" references. This standardless method allows for rapid assessment of a material but should be evaluated by analysis of known multielement standards to determine the accuracy of the approach.

EDS spectra typically exhibit peak overlaps such as the well known interference of the Z-1 K $\beta$ peak on the $Z$ $\mathrm{K} \alpha$ peak, for example Ti $\mathrm{K} \beta$ overlapping $\mathrm{V} \mathrm{K} \alpha$. It is important to identify peaks from high energy to low energy and to determine that all peaks of an element are present in the spectrum. Some legendary mistakes have been made simply based on peak identification errors. For important samples it may be helpful to acquire spectra at different accelerating potential in order to confirm the element inventory. 
There are a number of public domain software tools that one can use to help understand EDS analysis. The DTSA-II program can be used to simulate EDS spectra from bulk, particle, and stratified samples, and can be used to compare measured spectra to those generated for a given set of elements so that a "second opinion" is available [2]. This program also includes Monte Carlo simulation of electron scattering for different geometries and can also be used to perform quantitative analysis using standards. EDS spectra simulated with DTSA-II can be used to discriminate pulse pileup artifacts in an actual spectrum because the simulated spectrum does not include these artifacts from the counting electronics. The CalcZAF program can be used to calculate a number of parameters including ZAF correction factors, and is helpful for understanding the relative magnitudes of $\mathrm{x}$-ray absorption, atomic number, and $\mathrm{x}$-ray fluorescence in a given sample [3]. CalcZAF can be used to determine the correct accelerating voltage for analysis of samples with highly absorbed x-rays and can be used to calculate the expected x-ray intensity emitted from selected materials and can be compared to measurements made on real-world samples.

Fig. 1 shows a comparison of EDS spectra acquired on the mineral Kakanui hornblende using a SiLi EDS detector with a Be window, a modern SDD detector with an ATW window, and a simulated spectrum generated by DTSA-II. All the spectra illustrate how the EDS detector can be used to identify a number of elements very quickly, so that the identification of this mineral is made much simpler. The Be window of a SiLi detector results in strong absorption of low energy x-rays but the spectrum required 1000s to detect Mn. It shows relatively superior performance of the counting electronics for high energy x-rays; this is because the electronics are not spending time processing many low energy photons. The SDD spectrum was acquired in about $10 \%$ the time required for the SiLi spectrum and has obtained the same information. Note the pulse pileup artifact that could be interpreted as a peak for phosphorous, this is due to the simultaneous counting of carbon and silicon x-rays by the processing electronics. Finally, the simulated spectrum shows that the element inventory and EDS peaks are as expected with the exception of a carbon peak which is present on the SDD spectrum due to the carbon coat (that was not included in the DTSA-II simulation).

A number of examples of EDS spectra, artifacts, and issues regarding quantitative analysis will be presented during the presentation.

\section{References}

[1] Goldstein et. al., Scanning Electron Microscopy and X-ray Microanalysis, Springer, 2003.

[2] http://www.cstl.nist.gov/div837/837.02/epq/dtsa2/index.html

[3] http://epmalab.uoregon.edu/calczaf.htm

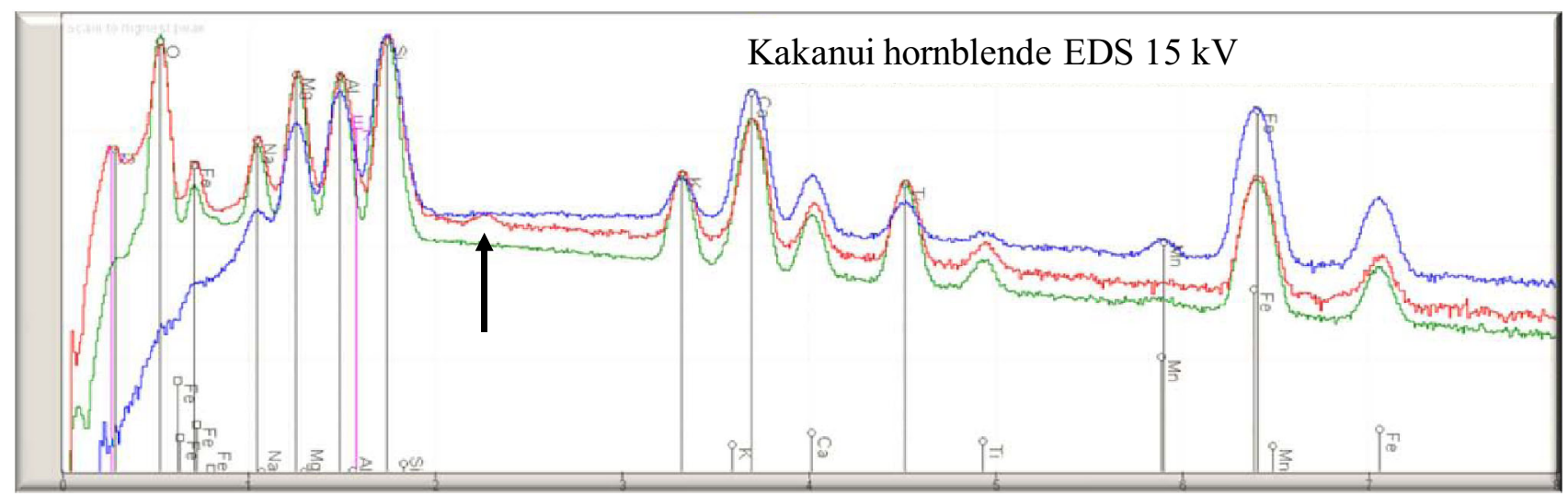

Figure 1 EDS spectra acquired on Kakanui hornblende: carbon-coated and polished bulk specimen at $15 \mathrm{kV}$ and 40 degree takeoff, displayed in $\log$ scale for counts and 0-8 kV horizontal axis. Blue: SiLi detector with 7 mil Be window showing significant low energy absorption but good sensitivity for higher energy x-rays $(\mathrm{Mn} \mathrm{K} \alpha)$. Red: SDD detector with ATW thin window showing excellent low energy performance but pulse pileup (arrow) and continuum infill (e.g., between Fe K $\alpha$ and K $\beta$ ). Green: DTSA-II simulated spectrum, indicating a need to adjust ATW window parameters to better match low energy portion of SDD spectrum. 